\title{
Predictive modelling of Ross River virus notifications in southeastern Australia
}

\author{
Z. CUTCHER ${ }^{1,2}$, E. WILLIAMSON ${ }^{1,3}$, S. E. LYNCH ${ }^{4}$, S. ROWE ${ }^{2}$, \\ H. J. CLOTHIER ${ }^{1}$ AND S. M. FIRESTONE ${ }^{5 *}$ \\ ${ }^{1}$ Melbourne School of Population and Global Health, The University of Melbourne, Parkville, Victoria, Australia \\ ${ }^{2}$ Victorian Department of Health and Human Services, Communicable Disease Epidemiology and Surveillance, \\ Health Protection Branch, Melbourne, Victoria, Australia \\ ${ }^{3}$ London School of Hygiene and Tropical Medicine, London, UK \\ ${ }^{4}$ Victorian Department of Economic Development, Jobs, Transport and Resources, Biosciences Research \\ Division, AgriBio Centre, Bundoora, Victoria, Australia \\ ${ }^{5}$ Asia-Pacific Centre for Animal Health, Faculty of Veterinary and Agricultural Sciences, The University of \\ Melbourne, Parkville, Victoria, Australia
}

Received 20 January 2016; Final revision 4 September 2016; Accepted 13 October 2016; first published online 21 November 2016

\section{SUMMARY}

Ross River virus (RRV) is a mosquito-borne virus endemic to Australia. The disease, marked by arthritis, myalgia and rash, has a complex epidemiology involving several mosquito species and wildlife reservoirs. Outbreak years coincide with climatic conditions conducive to mosquito population growth. We developed regression models for human RRV notifications in the Mildura Local Government Area, Victoria, Australia with the objective of increasing understanding of the relationships in this complex system, providing trigger points for intervention and developing a forecast model. Surveillance, climatic, environmental and entomological data for the period July 2000-June 2011 were used for model training then forecasts were validated for July 2011-June 2015. Rainfall and vapour pressure were the key factors for forecasting RRV notifications. Validation of models showed they predicted RRV counts with an accuracy of $81 \%$. Two major RRV mosquito vectors (Culex annulirostris and Aedes camptorhynchus) were important in the final estimation model at proximal lags. The findings of this analysis advance understanding of the drivers of RRV in temperate climatic zones and the models will inform public health agencies of periods of increased risk.

Key words: Arboviruses, modelling, notifiable infectious diseases, surveillance.

\section{INTRODUCTION}

Ross River virus (RRV) (family Togaviridae, genus Alphavirus), is the most common mosquito-borne virus in Australia, with the largest burden occurring

\footnotetext{
* Author for correspondence: Dr S. M. Firestone, Asia-Pacific Centre for Animal Health, Faculty of Veterinary and Agricultural Sciences, The University of Melbourne, Parkville, Victoria 3010, Australia.

(Email: Simon.Firestone@unimelb.edu.au)
}

in the tropical north [1]. Symptoms in humans include debilitating fatigue, muscle and joint pain that persist between 3-6 months, and up to a year in some cases [2], leading to significant morbidity and economic loss [3]. However, $55-75 \%$ of cases are asymptomatic [4].

In the southeastern State of Victoria, RRV is endemic with seasonal incidence. Most cases occur during the Southern Hemisphere summer and early autumn, so reporting of arbovirus notifiable disease 
surveillance data typically refers to Australian financial years ( 1 July to 30 June the following calendar year) [1]. In the period July 2005-June 2010, a mean of 214 human cases were notified per year in Victoria (3.8/100 000 people per year), with the majority acquiring infection in either northern regions of the State (the Murray Valley) or southeastern coastal regions [1]. Outbreaks occurred in 1992/1993, 1996/ 1997, and more recently in 2010/2011 when 1312 cases were notified across the State $(23 \cdot 3 / 100000$ people) [5].

The epidemiology of RRV is complex with the disease maintained in wildlife reservoirs and transmitted to humans by mosquitoes, with human-mosquitohuman transmission potentially occurring during epidemics [4]. The virus has been isolated from over 40 different mosquito species; however, only a small number are thought to be competent vectors [6]. The predominant mosquito vector species vary by location and season. Macropods are thought to be the major wildlife reservoir, which also vary by ecological niche. Other marsupials, rodents and flying foxes may also be involved [6], particularly in urban areas [4]. Horses can also be clinically infected [7]; however, their role in amplifying the virus is unclear.

\section{Arboviral surveillance and intervention in Victoria}

RRV is a notifiable human disease under the Public Health and Wellbeing Regulations (2009). In Victoria, doctors and/or pathology laboratories must notify all laboratory-confirmed cases to the Department of Health and Human Services (DHHS) within 5 days of diagnosis. According to the nationally agreed case definition [1] laboratory definitive evidence confirming a case requires either:

- isolation of RRV, or

- detection of RRV nucleic acid, or

- immunoglobulin $\mathrm{G}(\mathrm{IgG})$ seroconversion or a significant increase in antibody level or a $\geqslant$ fourfold rise in titre to RRV, or

- detection of RRV-specific IgM, in the absence of Barmah Forest virus IgM, unless RRV $\operatorname{IgG}$ is also detected, or

- detection of RRV-specific IgM in the presence of RRV IgG.

Control of arboviruses relies on early detection of increased levels of mosquitoes and/or virus activity, prompting public health interventions including vector control and public education for bite prevention
[8]. Under the Victorian Arbovirus Disease Control Program (VADCP) local governments across Victoria implement surveillance and control strategies on vector mosquito populations during the peak season between November and April each year when most human arbovirus notifications are received [9]. This programme has been providing standardized adult mosquito monitoring and sentinel chicken surveillance targeted at Murray Valley encephalitis (MVE) and other endemic arboviruses since 1991 in a One Health model of collaboration. The Victorian Department of Economic Development, Jobs, Transport and Resources (DEDJTR) provides virological and entomological support to the VADCP, funded equally by the DHHS and the local governments involved, overseen by a multidisciplinary Task Force. Surveillance involves weekly mosquito trapping using carbon dioxide and light-baited traps in eight local government areas across Victoria. Mosquitoes are counted and identified by species and viral isolation is attempted in an effort to detect the presence of RRV.

Before and during each peak season for arboviral activity, the VADCP analyses three broad environmental indicators [9-11] of conditions suitable for increased MVE virus activity in southeastern Australia. Meteorological data [rainfall in the catchment basins of the four main river systems in eastern Australia and proxy measures for the Southern Oscillation Index (SOI) and La Niña events] are considered by DHHS and councils to inform of likely disease occurrence and when to instigate interventions. No models are currently available to combine these data for RRV prediction, with public health interventions being informed by routine notifiable disease surveillance and mosquito monitoring through the VADCP.

\section{Modelling and prediction}

Due to the climatic dependence of wildlife and mosquito populations, models using climate and/or entomological variables to predict RRV incidence may be helpful for informing disease control activities and forecasting the impact of climate change. A detailed review [3] describes previous models for RRV. Most predictive models for RRV have used logistic regression to estimate the odds or probability of an outbreak within a season, using seasonal variables at fixed points in time [12-16]. Others have explored prediction of disease using time-series analysis techniques [12], such as seasonal autoregressive integrated moving average and polynomial distributed 


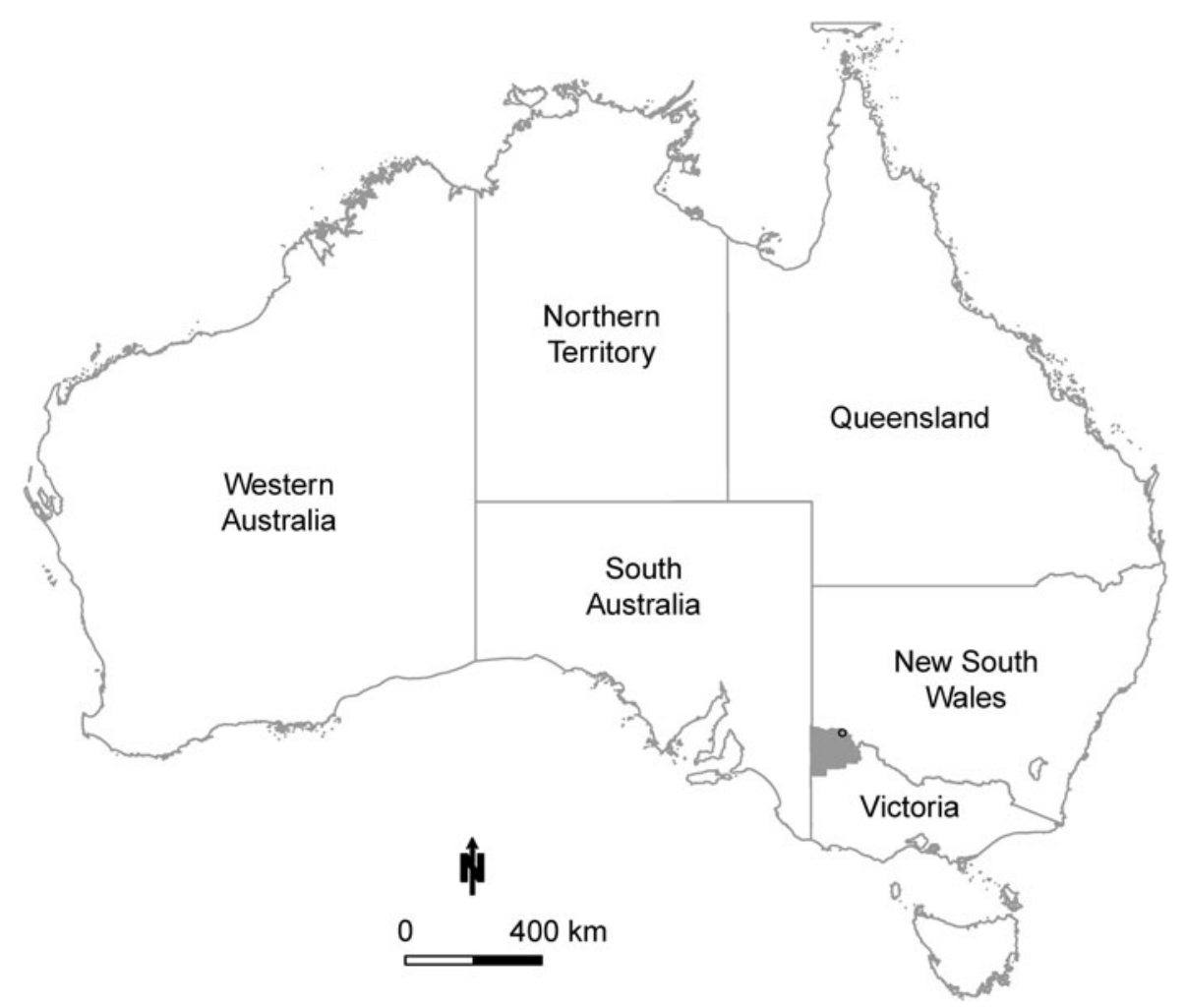

Fig. 1. Study extent of predictive modelling of Ross River virus cases in the Mildura Local Government Area (LGA) (shaded grey), Victoria, Australia, for the period 1 July 2000 to 30 June 2015. The black circle represents the location of the Mildura airport weather station. The Murray River forms the northern border of the Mildura LGA.

lag (PDL) time-series models [17], and also negative binomial regression [18], to predict rates of disease, rather than simply whether or not an outbreak might occur in a season. Models tailored to conditions at the local level have tended to have better predictive capacity than broader geographical models [13]. All previous models based on RRV surveillance data for southern Australia have estimated associations with annual case counts, with only two incorporating both entomological and climatic variables (for the southwestern region of Western Australia [13] and southern South Australia [15]). None of the models for RRV in southern Australia have attempted to model monthly counts and none have explicitly undertaken out-ofsample validation (forecasting); however, their outputs have informed surveillance and control activities.

Models combining mosquito count and climate data have produced better results than models considering climatic variables alone [13, 17]. For example, Woodruff et al. [13] developed early and late warning models for RRV outbreak years in 14 statistical local areas of Western Australia and found climate data alone had $64 \%$ sensitivity for an early warning model, and the addition of mosquito surveillance data increased the sensitivity to $85 \%$. Previous models for predicting RRV in Victoria [16] have used only climatic data at one time point per season (total rainfall in July, maximum temperature in November) to estimate the probability of an outbreak during peak transmission season for two adjacent areas in the Murray Valley, achieving in-sample sensitivity (internal 'rotational' validation) of between $64 \%$ and $96 \%$ for predicting an outbreak season.

The aim of this analysis was to develop predictive models for monthly counts of human RRV notifications in a highly affected inland location. Specific objectives included estimating the association between notified case counts and explanatory climatic, environmental and entomological variables, evaluating the usefulness of mosquito count data for informing public health interventions by estimating trigger points for action and, last, developing a forecasting tool.

\section{METHODS}

\section{Data}

Mildura Local Government Area (LGA), located inland in northwest Victoria (Fig. 1) was selected for 
Table 1. Climatic and environmental variables tested as predictors in models of monthly Ross River virus notifications for the Mildura Local Government Area, Victoria, Australia

\begin{tabular}{|c|c|c|c|}
\hline Variable & Description & Unit & Source \\
\hline SOI & Southern Oscillation Index (monthly) & - & $\begin{array}{l}\text { Australian Bureau of } \\
\text { Meteorology }\end{array}$ \\
\hline RAIN & Total monthly precipitation & $\mathrm{mm}$ & \\
\hline RAINDAYS & Number of days with $>1 \mathrm{~mm}$ precipitation per month & days & \\
\hline TMAX & Absolute maximum air temperature per month & ${ }^{\circ} \mathrm{C}$ & \\
\hline TMIN & Absolute minimum air temperature per month & ${ }^{\circ} \mathrm{C}$ & \\
\hline TDMEAN & Mean daily air temperature per month & ${ }^{\circ} \mathrm{C}$ & \\
\hline TDMAX & Mean daily maximum air temperature per month & ${ }^{\circ} \mathrm{C}$ & \\
\hline TDMIN & Mean daily minimum air temperature per month & ${ }^{\circ} \mathrm{C}$ & \\
\hline HUM & $\begin{array}{l}\text { Humidity at } 9 \text { a.m. on the day with the maximum } \\
\text { temperature per month }\end{array}$ & $\%$ & \\
\hline VAP & Maximum vapour pressure at 9 a.m. per month & $\mathrm{hPa}$ & \\
\hline VAPS & Maximum saturated vapour pressure at 9 a.m. per month & $\mathrm{hPa}$ & \\
\hline SST & Monthly sea surface temperature (SST) and sea surface & ${ }^{\circ} \mathrm{C}$ & National Oceanic and \\
\hline SSTA & $\begin{array}{l}\text { temperature anomaly (SSTA) measured at Niño } 3 \cdot 4 \\
\text { (a standardized region for sea surface temperature } \\
\text { measurement in the Pacific Ocean) }\end{array}$ & & $\begin{array}{l}\text { Atmospheric } \\
\text { Administration }\end{array}$ \\
\hline SEALVL $_{\min }$ & Monthly minimum, maximum and mean sea level measured & M & National Tidal Centre \\
\hline SEALVL $_{\max }$ & by tide height at Stony Point, Victoria, Australia & & \\
\hline SEALVL $_{\text {mean }}$ & $\left(\right.$ GDA94: $\left.38 \cdot 373155^{\circ} \mathrm{S}, 145 \cdot 223538^{\circ} \mathrm{E}\right)$ & & \\
\hline RIVER & $\begin{array}{l}\text { Maximum daily river flow for the Murray River at Colignan } \\
\text { (indicative of irrigation) }\end{array}$ & ML & $\begin{array}{l}\text { Victorian Water Resources } \\
\text { data warehouse }\end{array}$ \\
\hline
\end{tabular}

this analysis as it has the highest RRV disease burden in the State. RRV notifiable disease surveillance data for the period July 2000-June 2015 were provided by the DHHS including the following variables: estimated date of onset, 5-year age groups, sex and residential address (or exposure address where ascertained at interview by health officials). These data were geocoded utilizing the Google Maps ${ }^{\circledR}$ application programming interface, aggregated by month of onset and divided by annual Australian Bureau of Statistics estimates of the resident LGA population.

Weekly mosquito trapping count data were provided by DEDJTR for the same time period, for four traps in the Mildura LGA. Six species of interest were investigated for predictive value, including two thought to play a major role in Victoria in RRV transmission [4] (Aedes camptorhynchus and Culex annulirostris), two mosquito species with possible roles in transmission (Ae. notoscriptus, Coquillettidia linealis) and two further species with unknown importance for RRV transmission (Cx. australicus, a vector of MVE, and $C x$. globicoxitus). Mosquitoes are only counted during November-April each year. The median, mean and maximum counts across the four traps located in the Mildura LGA were calculated each month and categorized as follows for each species: 'no mosquitoes trapped' (the reference category), '1-9 mosquitoes', '10-99 mosquitoes', '100-999 mosquitoes', and ' $\geqslant 1000$ mosquitoes'.

Climatic and environmental variables were selected following a review of previous models, and are summarized by source in Table 1 . Weather station data were obtained from the Australian Bureau of Meteorology weather station with the most complete data in Mildura LGA (Mildura airport; Bureau of Meteorology Station no.: 076031; geo-coordinates $142 \cdot 0867^{\circ} \mathrm{E},-34 \cdot 2358^{\circ} \mathrm{S}$, see Fig. 1).

\section{Descriptive and univariable statistical analyses}

The distribution of each variable was examined and described, using contingency tables for categorical variables, collapsing categories where appropriate. Summary statistics and histograms were inspected for continuous variables and these transformed as required.

Data for the period July 2000-June 2011 were used to train the model. Owing to overdispersion, negative binomial regression models were constructed to predict the monthly count of notified RRV cases each 
month for Mildura LGA (y), of the form:

$Y \sim \operatorname{Poisson}\left(\mathrm{mu}^{*}\right)$,

$\ln \left(\mathrm{mu}^{*}\right)=\beta_{0}+\beta_{1} x_{1}+\beta_{2} x_{2}+\ldots+\beta_{p} x_{p}+v$,

$\exp (v) \sim \operatorname{Gamma}\left(\frac{1}{\alpha}, \alpha\right)$,

where the $p$ predictor variables $x_{1}, x_{2}, \ldots, x_{p}$ are given, and the population regression coefficients $\beta_{0}, \beta_{1}, \ldots, \beta_{p}$ are estimated, applying a dispersion parameter $(\alpha)$ to represent the ratio of the variance of the expected counts to their mean. The dispersion parameter affects the variance of the expected counts, not the expected counts themselves. Exponentiation allows expression of the coefficients as incidence rate ratios (IRRs).

Climatic and entomological variables were lagged by $1-12$ months and screened for entry into multivariable modelling. For each putative predictor variable, the lag with the strongest statistical association was selected using Akaike's Information Criterion (AIC) [19] - as this criterion may be applied to non-nested models - and entered into multivariable models if they were crudely statistically associated with RRV case count based on a liberal $P$ value threshold $(P<$ $0 \cdot 25$ ). The linearity of the univariable relationship with the outcome variable was assessed graphically for each continuous variable and by comparing the AIC of univariable models including a linear term $v s$. those with the variable categorized into quintiles. Where appropriate categorized variables were retained for further analyses and category levels collapsed.

All continuous covariates were tested for collinearity in pairs by calculating Spearman's correlation coefficient $\left(\rho_{s}\right)$. Among pairs of highly correlated predictors $\left(\rho_{s} \geqslant 0 \cdot 70\right)$, only the variable with the strongest statistical association with the outcome was retained for further analysis [20].

\section{Multivariable analyses}

Multivariable models were constructed including all retained variables and trimmed for parsimony using manual backwards-stepwise regression to $P<0 \cdot 20$.

Each removed variable was re-entered individually into the preliminary main effects model and retained if $P<0 \cdot 15$. At this point, pairwise interactions were tested among all retained terms, categorizing continuous variables as required, and the model was reconstructed as a generalized linear model to implement regression diagnostics (deviance-based goodnessof-fit to the training data, assessment of residuals, influence and leverage). Maximum likelihood $R^{2}$ was used as a robust measure of fit (no universally accepted adjusted $R^{2}$ measure is available for negative binomial models [21]). The final 'estimation' model was checked for serial autocorrelation (AC) by including case counts in immediately preceding months [22] after testing for non-stationarity and trend in the time-series following the Dickey-Fuller (DF) approach [23].

\section{Prediction, validation and adjustment for overfitting}

The final estimation model was used to predict monthly notified human RRV case counts notified in each month in the 4-year validation dataset (July 2011-June 2015) for Mildura LGA, and 95\% prediction intervals (PIs) were estimated adapting the method of Farrington et al. [24] to the negative binomial distribution. External ('out-of-sample') forecasts and their 95\% PIs were then compared to observed data (not used in model development) using Pearson's correlation coefficient $\left(\rho_{p}\right)$ [25], and models were tested for their proportional agreement with subjectively defined 'outbreak alerts' (months with >2 notified cases and where the count of cases exceeded the 5 -year mean plus 1 s.D. for that month estimated excluding known outbreak years, i.e. 2010/2011, assuming a negative binomial distribution) [26]. The final estimation model was pruned to account for overfitting by removing variables sequentially, and the comparisons repeated, to arrive at the final 'prediction' model, selected based on its forecasting ability.

Analyses were undertaken using Stata v. 14.0 (StataCorp., USA) and the R statistical package v. 3.1.1 [27] using the libraries 'MASS' [28] and 'epiR' [29].

\section{RESULTS}

There were 479 notified cases of RRV in Mildura LGA during the study period. The outbreak during the 2010/2011 financial year accounted for 251 notifications $(52 \cdot 4 \%)$ (Fig. 2). The mean notification rate (excluding 2010/2011) was 63.9/100 000 person years $(32 \cdot 6 / 100000)$. Cases were notified year-round however $87 \%$ had estimated dates of onset between November and April. There were 31 outbreak alerts in the study period, six of these in 2010/2011 and 16 in the model validation period. 


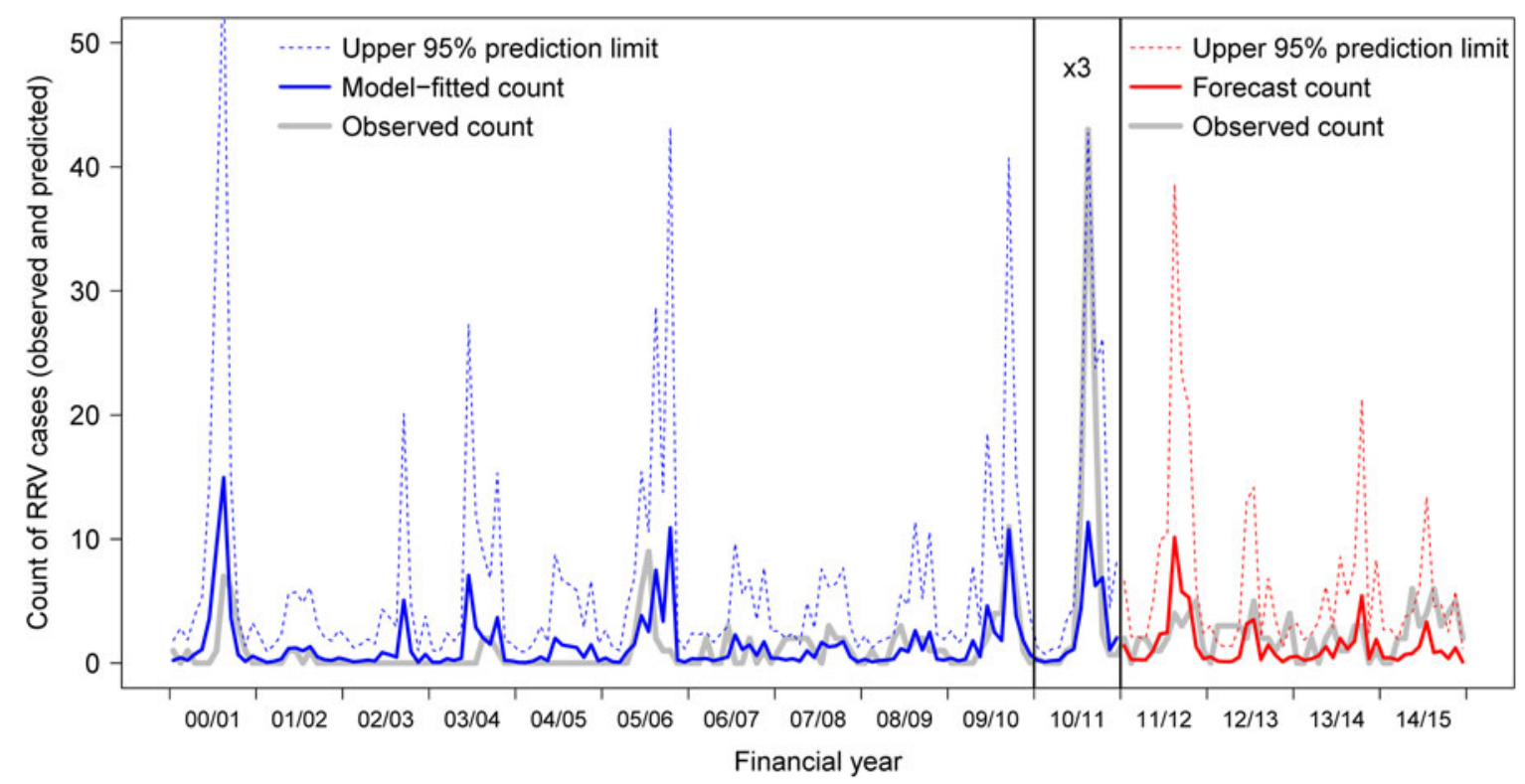

Fig. 2. Monthly time-series, predictions and forecasts of notified Ross River virus cases in the Mildura Local Government Area, Victoria, Australia, for the period 1 July 2000 to 30 June 2015. Data for the Australian financial year 2010/2011 have been rescaled by a factor of 3 . Dotted lines represent upper $95 \%$ prediction intervals.

Among those species investigated, the predominant mosquito species trapped in Mildura LGA during the study period were $C x$. annulirostris $(n=142638)$, Ae. camptorhynchus $(n=24349), C x$. australicus $(n=$ $6768)$ and Coquillettidia linealis $(n=5249)$. Univariable associations between RRV incidence in Mildura LGA and lagged counts of the mosquito species and climatic and environmental variables are provided in Supplementary Tables S1 and S2).

The final estimation model for Mildura LGA is presented in Table 2. A doubling of maximum vapour pressure was associated with a $3 \cdot 5$-fold rise in the rate of notifications in the following month (IRR $3 \cdot 47,95 \%$ CI $1 \cdot 57-7 \cdot 66)$. Mean trap counts of $C x$. annulirostris $\geqslant 1000$ were associated with a sevenfold increase in the rate of RRV notifications in the following month. When the mean Ae. camptorhynchus count was $\geqslant 10$, RRV notifications 2 months later were increased 55\%. A doubling of precipitation and more rain days, were associated with $25 \%$ and $8 \%$ rises in RRV notifications, 4 and 6 months later, respectively. Two interaction terms were retained in the final model. The main effect of Murray River flows in the highest quintile (maximum daily flow in a month $\geqslant 16268 \mathrm{ML}$ ) was an $85 \%$ reduction in RRV notifications 3 months later (IRR 0.15, 95\% CI $0 \cdot 03-0 \cdot 81$ ), whereas when the SOI (measured 6 months prior) was greater than its median across the study period ( $\geqslant 1.7$ units) Murray River flows in the highest quintile were associated with a 5.7-fold increase in the rate of RRV notifications 3 months later. The main effect of Pacific Ocean sea surface temperatures $\geqslant 26.8{ }^{\circ} \mathrm{C}$ was a $68 \%$ reduction in notifications 2 months later, whereas when minimum monthly sea levels (measured 7 months prior) were $\geqslant 13.2 \mathrm{~cm}$ and sea surface temperatures $\geqslant 26.8^{\circ} \mathrm{C}$ were associated with a fourfold rise in RRV notifications 2 months later.

There was no long-term trend in the time-series $(P=0 \cdot 14)$ and the null hypothesis of non-stationary was rejected (DF test statistic $=-5 \cdot 856$, D.F. $=132$, $P<0 \cdot 001$ ). Moderate serial AC was detected (lag 1, $\mathrm{AC}=0.61$ ) with each case 1 month prior being associated with a $12 \%$ increase in RRV incidence the following month (IRR $1 \cdot 12,95 \%$ CI 1.05-1·19). An AC term was included then eliminated (owing to $P>0 \cdot 20$ ) from the final estimation model.

Forecast ability of the model was improved by pruning to the final forecasting model (presented in Table 3 with a comparison of observed data and forecasts). Total observed annual counts were within forecast prediction intervals in all four validation years (Fig. 2), and at a monthly resolution observed counts were within the forecast prediction intervals in 39 of 48 months in the validation period $(81 \%)$, compared to 129 of 132 months in the model training period $(98 \%)$. In two of the validation years (2011/2012 and 2013/2014) there was excellent agreement between 
Table 2. Final negative binomial regression ('estimation') model for monthly Ross River virus notifications in the Mildura Local Government Area, Victoria, Australia, July 2000-June 2011

\begin{tabular}{|c|c|c|c|c|c|c|}
\hline Variable (units) & $\begin{array}{l}\text { Lag } \\
\text { (months) }\end{array}$ & Levels & IRR & S.E. & $95 \% \mathrm{CI}$ & $P$ value \\
\hline $\log _{2}($ maximum vapour pressure, $\mathrm{hPa})$ & 1 & & $3 \cdot 47$ & $1 \cdot 40$ & $1 \cdot 57-7 \cdot 66$ & $0 \cdot 002$ \\
\hline \multirow[t]{3}{*}{ Culex annulirostris (mean trap count) } & 1 & $\geqslant 1000$ & $7 \cdot 07$ & $3 \cdot 37$ & $2 \cdot 78-18 \cdot 0$ & $<0 \cdot 001$ \\
\hline & & $10-999$ & $1 \cdot 83$ & $0 \cdot 46$ & $1 \cdot 12-2 \cdot 98$ & \\
\hline & & $<10$ & $1 \cdot 00$ & - & (ref.) & \\
\hline \multirow[t]{2}{*}{ Aedes camptorhynchus (mean trap count) } & 2 & $\geqslant 10$ & $1 \cdot 55$ & $0 \cdot 23$ & $1 \cdot 16-2 \cdot 09$ & $0 \cdot 003$ \\
\hline & & $<10$ & $1 \cdot 00$ & - & (ref.) & \\
\hline $\log _{2}($ precipitation, $\mathrm{mm})$ & 4 & & $1 \cdot 25$ & $0 \cdot 08$ & $1 \cdot 09-1 \cdot 42$ & $0 \cdot 001$ \\
\hline Number of days with precipitation $>1 \mathrm{~mm}$ & 6 & & $1 \cdot 08$ & $0 \cdot 04$ & $1 \cdot 00-1 \cdot 17$ & $0 \cdot 044$ \\
\hline \multicolumn{7}{|l|}{ Interaction terms } \\
\hline \multirow{2}{*}{\multicolumn{7}{|c|}{$\begin{array}{l}\text { Maximum Murray river flow } \times \text { Southern } \\
\text { Oscillation Index }\end{array}$}} \\
\hline & & & & & & \\
\hline RIVER & 3 & $\geqslant 16268 \mathrm{ML}$ & $0 \cdot 15$ & $0 \cdot 13$ & $0 \cdot 03-0 \cdot 81$ & $0 \cdot 027$ \\
\hline \multirow[t]{3}{*}{ SOI } & 6 & $\geqslant 1 \cdot 7$ & $1 \cdot 56$ & $0 \cdot 50$ & $0 \cdot 83-2 \cdot 94$ & $0 \cdot 167$ \\
\hline & & $-5 \cdot 4-1 \cdot 7$ & 1.99 & $0 \cdot 66$ & $1 \cdot 04-3 \cdot 80$ & $0 \cdot 037$ \\
\hline & & $<-5 \cdot 4$ & $1 \cdot 00$ & - & (ref.) & \\
\hline \multirow[t]{2}{*}{ RIVER $\times$ SOI } & & $\geqslant 1 \cdot 7$ & $5 \cdot 65$ & $4 \cdot 69$ & $1 \cdot 11-28 \cdot 7$ & $0 \cdot 037$ \\
\hline & & $-5 \cdot 4-1 \cdot 7$ & $0 \cdot 33$ & $0 \cdot 42$ & $0 \cdot 03-4 \cdot 10$ & $0 \cdot 385$ \\
\hline \multicolumn{7}{|l|}{ Sea surface temperature $\times$ minimum sea level } \\
\hline SST & 2 & $\geqslant 26 \cdot 8^{\circ} \mathrm{C}$ & $0 \cdot 32$ & $0 \cdot 09$ & $0 \cdot 19-0 \cdot 55$ & $<0 \cdot 001$ \\
\hline $\mathrm{SEALVL}_{\min }$ & 7 & $\geqslant 13 \cdot 2 \mathrm{~cm}$ & $0 \cdot 87$ & $0 \cdot 25$ & $0 \cdot 49-1 \cdot 55$ & $0 \cdot 644$ \\
\hline \multirow[t]{2}{*}{$\mathrm{SST} \times \mathrm{SEALVL} L_{\min }$} & & & $3 \cdot 99$ & $1 \cdot 72$ & $1 \cdot 71-9 \cdot 29$ & $0 \cdot 001$ \\
\hline & & & Est. & S.E. & $95 \% \mathrm{CI}$ & $P$ value* \\
\hline Dispersion parameter $(\alpha)$ & & & $0 \cdot 04$ & $0 \cdot 03$ & $0 \cdot 01-0 \cdot 21$ & $0 \cdot 009$ \\
\hline
\end{tabular}

IRR, Incidence rate ratio; S.E., standard error of IRR or dispersion parameter; CI, confidence interval.; Est., point estimate of dispersion parameter.

$\mathrm{AIC}=318 \cdot 564, n=132$, degrees of freedom $=16, \log$ likelihood $(\operatorname{model}=-143 \cdot 282 ;$ null model $=-226 \cdot 598)$, maximum likelihood $R^{2}=0 \cdot 717$, deviance-based goodness-of-fit $(P=0 \cdot 50)$.

* $P$ value for dispersion parameter estimated using a likelihood ratio test that $\alpha$ is non-zero.

forecast and observed case counts and outbreak alerts, proportional agreement of 0.92 and 0.83 , respectively. The model under-predicted case counts in 2012/2013 and 2014/2015, all 9 months with observed counts above the forecast prediction interval occurred in these two years, resulting in poorer proportional agreement $(0.50$ in both cases) with observed outbreak alerts in these two years.

\section{DISCUSSION}

Climate, environmental and entomological variables were used to develop prediction models for monthly RRV incidence rates for the Victorian inland LGA with the highest notification rates. To our knowledge, this study was the first to integrate mosquito count data into Victorian RRV predictive modelling and the first to attempt out-of-sample forecasting of monthly counts of RRV for a location in southern Australia.
The most robust way to assess predictive model accuracy is to review a graphical representation of observed vs. predicted events using external data [30], as adopted for assessing the current models. The final forecasting model performed extremely well at tracking the observed counts in the validation period, and clearly fit the data well (differentiating between the outbreak year 2010/2011 and other years with relatively low counts). Forecast prediction intervals encompassed the observed monthly counts in 39 of 48 months in the validation period. Of the 9 months with observed counts falling above the predicted interval, five in 2012/2013 and two in 2014/ 2015 had very low notified case counts $(\leqslant 4)$ and raised outbreak alerts merely on the basis that these low counts were well outside the typical RRV activity season (when typically $\leqslant 1$ case was observed in most other years). The subjectively defined outbreak alert threshold is likely to be oversensitive, so direct 
Table 3. Final negative binomial regression ('forecasting') model for monthly Ross River virus notifications in the Mildura local government area, Victoria, Australia. Trained on data for the period July 2000-June 2011, validated on data for the period July 2012-June 2015

\begin{tabular}{llllll}
\hline \hline Variable (units) & Lag (months) & IRR & s.E. & $95 \%$ CI & $P$ value \\
\hline $\begin{array}{l}\log _{2} \text { (maximum vapour } \\
\text { pressure, hPa) }\end{array}$ & 1 & $13 \cdot 7$ & $6 \cdot 01$ & $5 \cdot 76-32 \cdot 4$ & $<0 \cdot 001$ \\
$\begin{array}{l}\log _{2} \text { (precipitation, mm) } \\
\begin{array}{c}\text { Number of days with } \\
\text { precipitation }>1 \mathrm{~mm}\end{array}\end{array}$ & 4 & $1 \cdot 44$ & $0 \cdot 13$ & $1 \cdot 21-1 \cdot 71$ & $<0 \cdot 001$ \\
& 6 & $1 \cdot 16$ & $0 \cdot 08$ & $1 \cdot 01-1 \cdot 33$ & $0 \cdot 036$ \\
Dispersion parameter $(\alpha)$ & & & & \\
Forecasting performance & & Est. & S.E. & $95 \%$ CI & $P$ value* \\
Financial year & Forecast cases & Observed & & & $0 \cdot 009$ \\
\hline $2011 / 2012$ & $(95 \%$ PI) & cases & $\rho_{P}$ & Forecast & Observed \\
$2012 / 2013$ & $30(0-122)$ & 27 & $0 \cdot 59$ & 2 & outbreak alerts \\
$2013 / 2014$ & $11(0-52)$ & 30 & $0 \cdot 32$ & 2 & 3 \\
$2014 / 2015$ & $19(0-88)$ & 16 & $0 \cdot 58$ & 1 & 6 \\
\hline \hline
\end{tabular}

IRR, Incidence rate ratio; s.E., standard error of IRR; CI, confidence interval; PI, prediction interval; $\rho_{P}$, Pearson's correlation coefficient.

$\mathrm{AIC}=367 \cdot 6142, n=132$, degrees of freedom $=5, \log$ likelihood ( $\operatorname{model}=-178 \cdot 807$; null model $=-226 \cdot 598)$, maximum likelihood $R^{2}=0 \cdot 515$, deviance-based goodness-of-fit $(P=0 \cdot 81)$.

* $P$ value for dispersion parameter estimated using a likelihood ratio test that $\alpha$ is non-zero.

comparisons can only be interpreted with caution. Raising the alert threshold to $>2$ s.D. than the longterm mean did not resolve the issue, as such a threshold was largely insensitive at detecting months that appeared to be clearly in excess of normal.

Statistical epidemiological modelling is often applied to address questions of causality (estimation and hypothesis testing) with fewer examples where the explicitly stated aim is modelling for prediction of future observations [22]. When forecasting (predicting into the 'out-of-sample' future), a modified approach may be required, as was the case in this study, reducing the focus on the relationships between individual variables. While model fit remains important there is a trade-off, external validity is paramount (models constructed based on historical data must hold into the near future) and overfitting to training data may well come at the expense of robust future prediction [22]. For this reason the final 'estimating' model, used for assessing the relationships between variables, was pruned to produce a more parsimonious 'forecasting' model.

Other models of RRV in southern Australia have been restricted to providing early warning of outbreak years, rather than attempting to forecast monthly counts. As presented, the forecasting model will be utilized each year to provide forecasts to the DHHS. Further modelling will be required to refine the variable selection and improve the robustness of forecasts. Other more complex approaches may be required [25], perhaps following the PDL modelling approach that $\mathrm{Hu}$ et al. [17] implemented for Brisbane, Queensland.

Rainfall and vapour pressure were key factors for forecasting RRV notifications in Mildura LGA. Rainfall has been included as an important predictor in all previous RRV models for southern Australia $[12,13,15,16]$, and underlies one of the broad early warning indicators [10] considered by DHHS for years of increased MVE activity. Vapour pressure is a measure of air humidity that depends on temperature and air pressure, similar variables have been included in all previous prediction models $[12,15$, 16] developed for regions along the Murray River (that forms a natural border between the states of Victoria and New South Wales). It is biologically plausible that these variables are related to arbovirus transmission, as mosquitoes require a minimum temperature and moisture for breeding. The lags of these variables likely reflect effects of water, temperature and climatic conditions on local ecology, for example through their effects on vegetation and wildlife reservoir host populations along with their direct 
effect on mosquito populations. While it is difficult to identify causal links between distally lagged precipitation variables and the timescales of vector development and transmission of RRV, the main purpose of the models developed here was as predictive tools rather than to draw explicit conclusions regarding causation. Including rainfall parameters with lags between 4 and 6 months provided the model with the best predictive performance at a monthly resolution. When we evaluated rainfall variables over lags of 1-3 months (in univariable analysis), very similar estimates were obtained as those included in the final model (for total monthly precipitation lagged 4 months, and number of days with $>1 \mathrm{~mm}$ rainfall lagged 6 months). There were only low levels of temporal AC observed between these variables, so these were included in multivariable estimation and prediction models at shorter lags (as secondary effects of rainfall over different time-scales). However, these variables representing shorter lags of rainfall were subsequently eliminated. Owing to weak correlations between climatic variables (rainfall, vapour pressure, humidity and temperature) in our data, it is also likely that some of the proximal effect of rainfall is represented by other variables in the final models.

$C x$. annulirostris and Ae. camptorhynchus are the two major mosquito vectors for RRV in Victoria [4]. Their inclusion in the final estimation model at proximal lags is consistent with their role in transmitting virus to humans from wildlife reservoirs and the time taken for mosquitoes to develop, the $\sim 2$ week extrinsic and 1- to 2-week intrinsic incubation periods of RRV [17]. The univariable associations presented in Supplementary Table S1 represent useful trigger points for action by the local council (such as mosquito larvicidal treatments and public announcements about the risk and appropriate preventative actions). Risk of RRV is likely to be greatly increased in months subsequent to those when mean weekly trap counts of Cx. annulirostris and Ae. camptorhynchus exceed 100 and 10 mosquitoes, respectively. Contrary to the findings of previous modelling studies of RRV notifications in other Australian states [13, 17], we found that inclusion of variables representing mosquito numbers provided no improvement in model forecasting ability (although strongly statistically significant associations were observed between lagged mosquito count variables and RRV notifications in the final estimation model). Hu et al. [17] noted the limitations of including mosquito count data in early warning forecasting models (cost of collection and proximal lags limiting the extent of early warning).

Two interesting interactions were present in the final estimation model, both of which appear indicative of periods of extreme climatic conditions. Elevated SOI (i.e. a La Niña event) 6 months earlier and maximum Murray River flow 3 months prior were associated with increased rates of notification for RRV. A severe flooding event affecting the Murray River valley occurred in the 2010/2011 outbreak year. Interestingly, on its own, high maximum Murray River flows (indicative of low amounts of irrigation) were associated with substantially decreased rates of RRV notification.

Weather patterns in the study region are heavily influenced by the development and intensity of El Niño/La Niña events in the Pacific Ocean [31]. Across eastern Australia, El Niño events are often associated with drier than normal conditions while La Niña events are associated with wetter than normal conditions. Lower sea surface temperatures in the Niño 3.4 region (SST) are an indicator of $\mathrm{La}$ Niña events and in this analyses were associated with increased rates of RRV notification, which is biologically plausible as wetter conditions favour mosquito larval development. Sea surface temperature was considered as a potential model covariate, even for this inland study area, as it was identified by Woodruff et al. [16] as a predictor in their model of RRV for the Murray region in Victoria, and for its role in the El Niño Southern Oscillation phenomenon that influences weather patterns across Australia.

Of interest, another biologically plausible and statistically significant interaction was detected, between SST and sea levels (when both were increased, rates of notification of RRV cases were also likely to be increased). Sea-level changes are driven by complex processes including thermal expansion of water, input of water into the ocean from glaciers and ice sheets, and changed water storage on land [32]. Variables representing sea level were considered for inclusion in these models because sea levels are correlated with SST and the SOI [33]. Again, this interaction term may indicate periods of extreme climatic conditions, with extremes in sea levels and sea surface temperature being a feature of cyclones (as experienced in the 2010/2011 outbreak year when cyclones in Queensland caused major flooding in the Murray-Darling river basin immediately preceding extremely high arbovirus activity). The DHHS utilizes another sea surface temperature measure, the Indian 
Ocean Dipole (IOD), which is based on the difference between sea surface temperature in the western and eastern tropical Indian Ocean, as a predictor for MVE virus activity in southeastern Australia [9]. Negative IOD events generally coincide with La Niña events.

The study was subject to a number of limitations: notification data may be undoubtedly understated and biased toward cases with typical clinical symptoms - those with less severe illness may not seek medical help or may be misdiagnosed. For this reason model outputs are interpreted as notification rates (rather than incidence rates). Residential location was accepted as a proxy for place of infection as this information was not available for a majority of cases. Misclassification of place of infection for some cases may have altered the measured associations between model covariates and disease, thus reducing predictive accuracy. The model did not account for mosquito control activities, as a reliable, consistent measure of these activities was unavailable. It is likely this omission has reduced the predictive accuracy of the models and ideally these should be accounted for in future research. Despite these limitations, the model presented appears a useful forecasting tool for RRV in region investigated with $81 \%$ of observed monthly counts in the validation period falling within forecast prediction intervals.

Changing climatic conditions over the coming decades are likely to alter the current patterns of arboviral disease in Australia [3,34], although the nature of this change is controversial [35]. The effect on arbovirus transmission is likely to vary regionally. For example, the impact will differ in arid compared to temperate, and coastal versus inland regions, reflecting variation in the effect of climate change on local ecological conditions [34]. Advanced tools, such as the models presented here, will be required to monitoring the changing relationship between notified cases and local conditions, and to provide early warning of periods of high arbovirus activity.

\section{SUPPLEMENTARY MATERIAL}

For supplementary material accompanying this paper visit https://doi.org/10.1017/S0950268816002594.

\section{ACKNOWLEDGEMENTS}

The authors gratefully acknowledge the following for preparing and providing data necessary for these analyses: the Victorian Government Department of Health and Human Services (particularly Nicola Stephens, Rebecca Feldman and Katherine Gibney), and the Department of Economic Development, Jobs, Transport and Resources (particularly Karen Brown, Joe Azuolas, Kim Andrews and Elwyn Wishart), the Mildura Rural City Council (Dale Hutchinson and Stuart Maher), the Victorian Arbovirus Taskforce and the Australian Bureau of Meteorology's National Climate Data Service. Human notifiable surveillance data was provided by the Department of Health and Human Services in accordance with its privacy act after ethical approval for all procedures used in this study was obtained from the Human Research Ethics Committee of the University of Melbourne (1339794).

This research received no specific grant from any funding agency, commercial or not-for-profit sectors. Dr Simon Firestone is supported by an Australian Research Council Discovery Early Career Researcher Award (project number DE160100477).

\section{DECLARATION OF INTEREST}

None.

\section{REFERENCES}

1. Knope $\mathbf{K}$, et al. Arboviral diseases and malaria in Australia, 2010-11: annual report of the National Arbovirus and Malaria Advisory Committee. Communicable Diseases Intelligence Quarterly Report 2013; 37: E1.

2. Westley-Wise VJ, et al. Ross River virus infection on the north coast of New South Wales. Australian and New Zealand Journal of Public Health 1996; 20: 87-92.

3. Jacups SP, Whelan PI, Currie BJ. Ross River virus and Barmah Forest virus infections: a review of history, ecology, and predictive models, with implications for tropical northern Australia. Vector-Borne and Zoonotic Diseases 2008; 8: 283-298.

4. Harley D, Sleigh A, Ritchie S. Ross River virus transmission, infection, and disease: a cross-disciplinary review. Clinical Microbiology Reviews 2001; 14: 909932.

5. Australian Government Department of Health. National notifiable diseases surveillance system (NNDSS). (http:// www9.health.gov.au/cda/source/cda-index.cfm) Accessed 11 February 2015.

6. Russell RC. Ross River virus: ecology and distribution. Annual Review of Entomology 2002; 47: 1-31.

7. El-Hage CM, McCluskey MJ, Azuolas JK. Disease suspected to be caused by Ross River virus infection of horses. Australian Veterinary Journal 2008; 86: 367-370.

8. van den Hurk AF, et al. Evolution of mosquito-based arbovirus surveillance systems in Australia. Journal of 
Biomedicine and Biotechnology 2012; volume 2012, Article ID 325659, 8 pp. doi:10.1155/2012/325659.

9. Lynch S, et al. Victorian Arbovirus Disease Control Program Annual Report 2014-2015. The State of Victoria Department of Economic Development, Jobs, Transport and Resources: Melbourne, 2015.

10. Forbes JA. Murray Valley Encephalitis 1974, Also, The Epidemic Variance Since 1914 and Predisposing Rainfall Patterns. Sydney: Australasian Medical Publishing Company, 1978.

11. Nicholls N. A method for predicting murray valley encephalitis in Southeast Australia using the Southern Oscillation. Australian Journal of Experimental Biology and Medical Science 1986. 64.

12. Bi P, et al. Defining the local ecology of Ross River virus infections as a basis for risk assessment and better prevention strategies in South Australia: final report prepared for the S.A. Department of Health. The University of Adelaide: Adelaide, 2006.

13. Woodruff RE, et al. Early warning of Ross River virus epidemics: combining surveillance data on climate and mosquitoes. Epidemiology 2006; 17: 569-575.

14. Gatton ML, Kay BH, Ryan PA. Environmental predictors of Ross River virus disease outbreaks in Queensland, Australia. American Journal of Tropical Medicine and Hygiene 2005. 72: 792-799.

15. Williams CR, Fricker SR, Kokkinn MJ. Environmental and entomological factors determining Ross River virus activity in the River Murray Valley of South Australia. Australian and New Zealand Journal of Public Health 2009; 33: 284-288.

16. Woodruff RE, et al. Predicting Ross River virus epidemics from regional weather data. Epidemiology 2002; 13: 384-393.

17. Hu W, et al. Rainfall, mosquito density and the transmission of Ross River virus: a time-series forecasting model. Ecological Modelling 2006; 196: 505-514.

18. Werner A, et al. Environmental drivers of Ross River virus in southeastern Tasmania, Australia: towards strengthening public health interventions. Epidemiology and Infection 2012; 140: 359-371.

19. Akaike H. Information theory and an extension of the maximum likelihood principle. In: Selected Papers of Hirotugu Akaike. New York: Springer, 1998, pp. 199-213.

20. Armitage P, Berry G, Matthews JNS. Statistical Methods in Medical Research, 4th edn. Oxford: WileyBlackwell, 2001, pp. 356-360.

21. Grimshaw JM, et al. Applying psychological theories to evidence-based clinical practice: identifying factors predictive of lumbar spine $\mathrm{x}$-ray for low back pain in UK primary care practice. Implementation Science 2011; 6: 55.

22. Dohoo I, Martin W, Stryhn H. Veterinary Epidemiologic Research, 2nd edn. Charlottetown, Prince Edward Island, Canada: VER Inc., 2009

23. Dickey DA, Fuller WA. Distribution of the estimators for autoregressive time series with a unit root. Journal of the American Statistical Association 1979; 74: 427-431.

24. Farrington C, et al. A statistical algorithm for the early detection of outbreaks of infectious disease. Journal of the Royal Statistical Society, Series A 1996; 1: 547563.

25. Axelsen JB, et al. Multiannual forecasting of seasonal influenza dynamics reveals climatic and evolutionary drivers. Proceedings of the National Academy of Sciences USA 2014; 111: 9538-9542.

26. Yohannes K, et al. Australia's notifiable diseases status, 2004, annual report of the National Notifiable Diseases Surveillance System. Communicable Disease Intelligence 2006; 30: 1-79.

27. R Core Team. R: A language and environment for statistical computing. $\mathrm{R}$ Foundation for Statistical Computing, Vienna, Austria. (http://www.R-project.org/). Accessed 15 September 2014.

28. Venables WN, Ripley BD. Modern Applied Statistics with S, 4th edn. New York: Springer, 2002.

29. Stevenson M, et al. epiR: an R package for the analysis of epidemiological data. $\mathrm{R}$ package version 0.9-69. 2015.

30. Moons KG, et al. Risk prediction models: I. Development, internal validation, and assessing the incremental value of a new (bio) marker. Heart 2012; 98: $683-690$.

31. Australian Government Bureau of Meteorology. El Niño - Detailed Australian Analysis (http://www.bom. gov.au/climate/enso/enlist/). Accessed 20 November 2015.

32. Rahmstorf S. A semi-empirical approach to projecting future sea-level rise. Science 2007; 315: 368-370.

33. Lombard A, et al. Contribution of thermal expansion to present-day sea-level change revisited. Global and Planetary Change 2005; 47: 1-16.

34. Woodruff RE, Bambrisk H. Climate change impacts on the burden of Ross River virus disease. In: Garnaut R, ed. Garnaut Climate Change Review. Port Melbourne: Cambridge University Press, 2008.

35. Russell RC. Mosquito-borne disease and climate change in Australia: time for a reality check. Australian Journal of Entomology 2009; 48: 1-7. 